\title{
Biliary Self-Expandable Metallic Stent Combined With lodine-125 Seeds in The Treatment of Malignant Biliary Obstruction (Bismuth Type I or II)
}

Kai-Cai Liu

USTC https://orcid.org/0000-0002-3561-7209

Wei-Fu Lv

USTC

Dong Lu ( $\sim$ ahslludong@163.com )

USTC

Chun-Ze Zhou

USTC

De-Lei Cheng

USTC

Shao-Bao Xu

USTC

Zong-Gen Gao

USTC

Chang-Sheng Shi

USTC: University of Science and Technology of China

Ming-Xue Su

USTC: University of Science and Technology of China

Research

Keywords: self-expandable metallic stent, malignant biliary obstruction,125I seeds

Posted Date: October 25th, 2021

DOI: https://doi.org/10.21203/rs.3.rs-962662/v1

License: (c) (i) This work is licensed under a Creative Commons Attribution 4.0 International License.

Read Full License 


\section{Abstract \\ Background}

Biliary self-expandable metallic stent combined with iodine- 125 seeds are increasingly used in patients with malignant biliary obstruction, rather than using percutaneous transhepatic cholangial drainage(PTCD) or biliary stents alone, because it has a good clinical effect.This study aimed to evaluate the safety and efficacy of a self-expandable metallic stent (SEMS) combined with iodine-125 ( $\left.{ }^{125} \mathrm{I}\right)$ seeds in the treatment of Bismuth type I or II malignant biliary obstruction (MBO).

\section{Methods}

The clinical data of 74 cases of MBO treated with percutaneous SEMS combined with ${ }^{125}$ I seeds (combination group) and 81 cases of MBO treated with SEMS implantation alone (control group) in our hospital from January 2015 to December 2019 were retrospectively analyzed.

\section{Results}

The surgical success rate of the two groups was $100 \%$, and no surgery-related deaths occurred. The liver function of each group was significantly improved after 1 week and 1 month of follow-up. There was no significant difference in short-term efficacy or complications between the two groups $(P>0.05)$. The average biliary opening times of the combined group and control group were $9.01 \pm 4.38$ months and $6.79 \pm 3.13$ months, respectively $(P<0.001)$. The average postoperative survival time was $12.08 \pm 5.38$ months and $9.10 \pm 4.16$ months, respectively $(P<0.001)$. Univariate and multivariate analyses showed that the type of biliary stent and liver metastasis were independent factors affecting survival.

\section{Conclusion}

SEMS combined with ${ }^{125} \mathrm{I}$ seeds is a safe, effective and feasible palliative treatment for patients with Bismuth type I or II MBO, as it can delay the recurrence of jaundice and prolong the survival time of patients.

\section{Introduction}

Malignant biliary obstruction (MBO) is directly or indirectly caused by malignant tumors such as pancreatic cancer and cholangiocarcinoma, with insidious onset and poor prognosis[1-2]. As tumor tissues tend to infiltrate surrounding tissues and organs, the overall surgical resection rate of tumor lesions is relatively low[3-4]. Even after surgery, complications such as liver failure, infection and tumor recurrence often occur. Biliary drainage and stent placement can quickly relieve the symptoms of biliary 
obstruction and improve the quality of life of patients, so they have become the preferred palliative treatment[5-6]. However, tumor tissue continues to grow after stent placement, which is likely to lead to stent reobstruction, and the long-term treatment effect is poor. According to one report, the patency time of bare metal biliary tract stents is approximately 88.3 days[7]. A fully covered metal biliary stent can effectively inhibit tumor tissue growth through the mesh and prolong the median patency time to approximately 118 days after stent implantation; however, the drainage of bile and pancreatic fluid may be affected after stent implantation, related complications may occur, and the displacement rate of these stents is higher[8]. If biliary stent implantation is combined with radionuclide internal radiotherapy, which has been widely used in clinical practice, these can complement each other and make up for each other's shortcomings. The stent can relieve symptoms of biliary obstruction and improve patients' systemic conditions. The radionuclide can inhibit tumor growth, improve the long-term patency rate of the stent and prolong the survival time. To perform radionuclide internal radiation therapy on or around a biliary stent, many domestic researchers have explored covering the biliary stent with a film coated with radionuclide particles[9]. ${ }^{125} \mathrm{I}$ seeds were directly bound to the biliary stent, or a plastic tube containing

${ }^{125}$ I seeds was pressed around the stent for treatment[4, 10-11]. However, the dose of radionuclides cannot be adjusted according to the characteristics of specific lesions in practical applications, and the consistency of the radiation dose and its distribution throughout the lesion cannot be guaranteed; the displacement, shedding and leakage of radionuclides after operation are also hidden dangers.

In this study, a brachytherapy system consisting of a particle carrying ${ }^{125}$ I seeds and a SEMS developed by Nanjing MicroPort, China, combined with a specially designed treatment planning system (TPS), was used in patients with MBO. This study aims to explore the safety and effectiveness of SEMS combined with ${ }^{125}$ I seed implantation in the biliary tract and whether it can prolong the biliary opening time and overall survival.

\section{Materials And Methods 2.1 Patients}

The data of patients with MBO who underwent biliary stenting in the First Affiliated Hospital of China University of Science and Technology from January 2015 to December 2019 were retrospectively analyzed.

The inclusion criteria were as follows: $\nabla$ age $18-80$ years; $\nabla$ clinical symptoms of biliary obstruction, such as jaundice; $\varangle$ malignant biliary obstruction confirmed by imaging, laboratory examination, tissue/cytology biopsy or previous operation; $₫$ Bismuth-Corlette type I or II bile duct obstruction; $\varangle$ Eastern Cooperative Oncology Group(ECOG) score of $0-3$; and $\triangle$ inability or refusal to undergo surgery. The

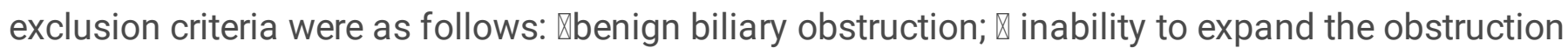

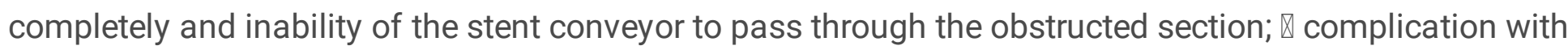
biliary perforation; $₫$ previous history of stent placement or biliary surgery; $\otimes$ contraindications of percutaneous transhepatic biliary drainage; $\triangle \mathrm{ECOG}$ score of 4 ; and $\otimes$ Bismuth-Corlette type III or IV biliary 
obstruction. Finally, according to different forms of biliary stents, the sample was divided into 81 patients receiving simple biliary stent treatment and 74 patients receiving internal irradiation with iodine-125 particles. The flow chart is shown in Fig. 1.

This study was approved by the Ethics Committee of the First Affiliated Hospital of the University of Science and Technology of China and was conducted in accordance with the standards of the Declaration of Helsinki. Due to the retrospective characteristics of this study, the ethics committee abandoned the requirement for informed consent.

\subsection{Device materials}

A percutaneous transhepatic biliary drainage (PTCD) trocar (Cook Medical, Bloomington, Indiana, USA), a supersmooth guide wire and a 5-F Cobra contrast catheter (Cordis, USA), etc. The biliary stent was provided by the Nanjing Institute of Minimally Invasive Research, China. The length of the stent was 6-10 $\mathrm{cm}$, and the diameter of the stent was $8-10 \mathrm{~mm}$. The structure of the stent was combined with particles is a double-layer structure: a SEMS and a stent carrying ${ }^{125}$ I seeds were deployed to the target bile duct and assembled. The ${ }^{125}$ I seeds were provided by Chengdu Yunke Company, China. The high-purity titanium shell was $0.8 \mathrm{~mm}$ in diameter and $4.5 \mathrm{~mm}$ in length. It had a built-in $3 \mathrm{~mm}{ }^{125} \mathrm{I}$ silver rod with an activity of $0.3 \mathrm{mCi}-6.0 \mathrm{mCi}$, a prescription dose of ${ }^{125}$ I seeds (CIAE- 6711; China Institute of Atomic Energy, Beijing). Planning and calculation were based on a treatment planning system (FTT technology, Beijing, China). Before the stent was placed, ${ }^{125}$ I seeds were assembled into the capsule of the stent, which was attached to the external surface of the stent.

\subsection{Operation method}

2.3.1 Imaging examination and preoperative evaluation: All patients underwent dual-phase computed tomography(CT) enhancement and Magnetic Resonance Cholangiopancreatography(MRCP) scanning to understand the tumor focus range, biliary obstruction level and length of stenotic segment and to determine the relationship between the tumor and the important vessels around it. This determines the length of the biliary tract particle scaffold, the location of implantation, and the number of ${ }^{125} \mathrm{I}$ seeds.

\subsubsection{Surgical operation:}

$\triangle$ With the patient in the supine position, we determined the skin puncture point and punctured the dilated bile duct with a PTCD special puncture needle under fluoroscopy or B-ultrasound monitoring. $\otimes$ We injected the contrast medium to show the length of the lesion and the degree of obstruction and mark them. We withdrew the catheter and replaced it with a $260 \mathrm{~cm}$ superhard and superlong guide wire. $\otimes W e$ chose an appropriate stent system for intrabiliary irradiation according to the length of the lesion. The

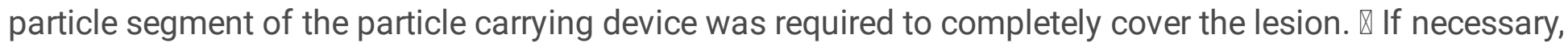
a balloon of appropriate length was used to dilate the obstructed biliary tract, and then the seeds carrying the device were pushed to the lesion site along the superhard guide wire. The proximal positioning method was used to confirm the accurate positioning before release. $\otimes$ After withdrawing the releaser, the 
common biliary stent was pushed to the biliary obstruction section along the superhard guide wire so that it overlapped with the particle segment of the biliary particle device. The upper and lower edges of the internal irradiation stent system had to exceed the lesion by approximately $10 \mathrm{~mm}$. $\nabla$ After the operation, the external drainage tube was put in, flushed, and clamped with metronidazole $50 \mathrm{ml}$ for 3 consecutive days. The drainage tube was left in for 1 week to understand the patency of the stent, and then the drainage tube was removed.

\subsubsection{Postoperative treatment:}

After operation, all patients received Electrocardiogram(ECG) monitoring, oxygen inhalation, liver protection, jaundice, hemostasis, anti-infection treatment, and symptomatic treatment. We observed the color, quality and quantity of the bile drainage fluid. For patients with unobstructed biliary drainage and no infection, bleeding or other signs, PTCD was clamped 3-7 days after operation according to the bile drainage volume, color and turbidity. If there was no obvious discomfort, the drainage tube was removed. One week after operation, a SPECT/CT scan was done to show the radioactivity and position of the ${ }^{125}$ I seed chain (Fig. 2). Interventional treatment was done to continue treatment of other lesions.

\subsection{Evaluation method and follow-up}

$\nabla$ Short-term efficacy: The incidence of postoperative complications was observed, and liver function, renal function and blood routine were reexamined 1 week and 1 month after operation to evaluate the recovery of liver function and the curative effect on jaundice regression.

Q Long-term efficacy: The long-term efficacy (postoperative biliary tract opening status and postoperative survival time) was compared between the observation group and the control group.

Q Influencing factors: To evaluate whether sex, age, etiology, liver function or obstruction site was an independent influencing factor of surgical efficacy, after the last return to the hospital for reexamination, telephone return visits were made once a month to gather data on the patient's survival, clinical symptoms, recurrence of jaundice and new complications. Three, 5, 7, 9 and 12 months after operation, CT was redone in the hospital or outside the hospital to view the distribution of the biliary stent and ${ }^{125}$ I seeds and evaluate the opening of the biliary tract.

\subsection{Statistical analysis}

SPSS22.0 statistical software (IBM, Armonk, NY, USA) was used for statistical analysis. Continuous data conforming to a normal distribution are presented as mean $\pm S D$ and were compared using Student's ttest. Continuous data that were not normally distributed are presented as median (interquartile range) and were compared using the Mann-Whitney U-test. The Kaplan-Meier method was used to analyze the stent opening time and survival time, and the log-rank test was used for comparison between groups. Multivariate Cox regression analysis was used to analyze the factors affecting surgical efficacy and survival. All results were statistically significant at $\mathrm{P}<0.05$. 


\section{Results}

\subsection{Surgical results}

The surgical success rate of all 155 patients was $100 \%$, The baseline characteristics of 81 patients receiving simple biliary stent treatment and 74 patients receiving biliary stent with $125 \mathrm{I}$ seeds treatment groups were well balanced.See Table 1. The survival time of all patients was more than 30 days, so they all could be evaluated for efficacy. 
Table 1

Baseline characteristics of two groups' patients

\begin{tabular}{|c|c|c|c|c|}
\hline Variables & Combination group $(n=74)$ & Control group $(n=81)$ & $x^{2} / t$ & $P$ value \\
\hline $\operatorname{Age}(\mathrm{Y})$ & & & 1.031 & 0.309 \\
\hline$\geq 50$ & 67 & 69 & & \\
\hline$<50$ & 7 & 12 & & \\
\hline Sex & & & 3.759 & 0.053 \\
\hline Male & 38 & 54 & & \\
\hline Female & 36 & 27 & & \\
\hline Bismuth-Corlette & & & 0.321 & 0.571 \\
\hline Type I & 35 & 42 & & \\
\hline Type II & 39 & 39 & & \\
\hline KFS(score) & & & 3.281 & 0.07 \\
\hline$\geq 70$ & 62 & 58 & & \\
\hline$<70$ & 12 & 23 & & \\
\hline \multicolumn{5}{|l|}{ Laboratory Test } \\
\hline TBIL $(\mu \mathrm{mol} / \mathrm{L})$ & $221.84 \pm 110.93$ & $214.48 \pm 148.83$ & 0.274 & 0.785 \\
\hline $\operatorname{ALT}(\mathrm{u} / \mathrm{L})$ & $106.99 \pm 81.02$ & $134.08 \pm 126.89$ & 1.228 & 0.222 \\
\hline $\mathrm{AST}(\mathrm{u} / \mathrm{L})$ & $113.85 \pm 80.10$ & $128.72 \pm 96.01$ & 0.842 & 0.402 \\
\hline $\mathrm{ALB}(\mu \mathrm{mol} / \mathrm{L})$ & $33.95 \pm 6.82$ & $35.03 \pm 5.53$ & 0.928 & 0.355 \\
\hline $\mathrm{PT}(\mathrm{s})$ & $11.95 \pm 1.83$ & $12.71 \pm 1.99$ & 1.796 & 0.051 \\
\hline Intrahepatic metastasis & & & 1.5 & 0.221 \\
\hline Yes & 32 & 43 & & \\
\hline No & 42 & 38 & & \\
\hline
\end{tabular}

PFS=Performance Status; $T B \|=$ total bilirubin;ALT=alanine aminotransferase; AST=aspartate aminotrans ferase; $A L B=$ albumin;PT=prothrombin time

\subsection{Short-term efficacy}

The liver function of all 155 patients improved significantly at 1 and 4 weeks after operation. Compared with preoperatively, the difference was statistically significant $(P<0.001)$; that is, the short-term effect of 
each treatment on malignant obstructive jaundice was good, and the jaundice obviously subsided. There was no significant difference between the two groups in the short-term effect $(P>0.05)$. See Table 2 . 
Table 2

.Laboratory Values before and after Stent Placement

\begin{tabular}{|c|c|c|c|}
\hline Laboratory Test & Combination group $(n=74)$ & control group $(n=81)$ & $P$ value \\
\hline \multicolumn{4}{|l|}{ TBIL (umol/L) } \\
\hline Preoperative & $214.48 \pm 148.83$ & $221.84 \pm 110.93$ & 0.785 \\
\hline After 1W & $141.87 \pm 92.47^{\#}$ & $151.68 \pm 93.95^{\#}$ & 0.707 \\
\hline After 1M & $64.40 \pm 64.08^{\#^{*}}$ & $69.35 \pm 63.55^{\#^{*}}$ & 0.782 \\
\hline$P$ & $P<0.001$ & $P<0.001$ & \\
\hline \multicolumn{4}{|l|}{ DBIL (umol/L) } \\
\hline Preoperative & $164.86 \pm 88.43$ & $178.65 \pm 63.39$ & 0.428 \\
\hline After 1W & $96.53 \pm 85.74^{\#}$ & $113.03 \pm 93.35^{\#}$ & 0.506 \\
\hline After $1 \mathrm{M}$ & $35.36 \pm 40.27^{\#^{*}}$ & $42.67 \pm 38.55^{\#^{*}}$ & 0.381 \\
\hline $\mathrm{p}$ & $P<0.001$ & $P<0.001$ & \\
\hline \multicolumn{4}{|l|}{ IBIL (umol/L) } \\
\hline Preoperative & $58.33 \pm 45.62$ & $50.68 \pm 43.39$ & 0.693 \\
\hline After $1 \mathrm{~W}$ & $45.34 \pm 25.38$ & $38.65 \pm 24.57$ & 0.436 \\
\hline After 1M & $29.04 \pm 25.38^{\#}$ & $26.38 \pm 18.43^{\#}$ & 0.212 \\
\hline$p$ & 0.001 & 0.152 & \\
\hline \multicolumn{4}{|l|}{$\operatorname{ALT}(\mathrm{u} / \mathrm{L})$} \\
\hline Preoperative & $106.99 \pm 81.02$ & $134.08 \pm 126.89$ & 0.222 \\
\hline After 1W & $68.54 \pm 48.61$ & $79.21 \pm 55.27$ & 0.426 \\
\hline After $1 \mathrm{M}$ & $32.36 \pm 14.72^{\#}$ & $42.65 \pm 27.62^{\#}$ & 0.182 \\
\hline$p$ & 0.021 & 0.047 & \\
\hline \multicolumn{4}{|l|}{ AST (u/L) } \\
\hline Preoperative & $106.21 \pm 84.45$ & $142.72 \pm 145.70$ & 0.425 \\
\hline After $1 W$ & $61.43 \pm 51.61^{\#}$ & $81.21 \pm 59.01$ & 0.354 \\
\hline After 1M & $30.29 \pm 13.94^{\#}$ & $47.86 \pm 29.78^{\#}$ & 0.056 \\
\hline$p$ & 0.005 & 0.031 & \\
\hline
\end{tabular}




\begin{tabular}{|llll|}
\hline Laboratory Test & Combination group $(\mathbf{n}=\mathbf{7 4})$ & control group $(\mathbf{n}=\mathbf{8 1})$ & $P$ value \\
\hline TBA(umol/L) & & & \\
\hline Preoperative & $56.44 \pm 33.24$ & $66.71 \pm 23.36$ & 0.454 \\
\hline After 1W & $43.67 \pm 34.40$ & $51.43 \pm 33.02$ & 0.056 \\
\hline After 1M & $27.33 \pm 14.50$ & $30.01 \pm 13.79^{\#}$ & 0.454 \\
$p$ & 0.439 & 0.013 & \\
\hline
\end{tabular}

\# Represents $\mathrm{P}<0.05$ compared with preoperatively, * represents $\mathrm{P}<0.05$ compared with one week after surgery. TBII=total bilirubin;ALT=alanine aminotransferase; $A S T=$ aspartate aminotrans ferase; DBIL=direct bilirubin; ILBI = indirect bilirubin; TBA =total bile acid;

\subsection{Complications}

The incidence of complications in the combined group was $18.92 \%$, including 8 cases of abdominal pain, 1 case of biliary bleeding, 2 cases of pancreatitis, 1 case of cholangitis and 2 cases of sepsis. The incidence of complications in the control group was $11.11 \%$, including 6 cases of abdominal pain, 2 cases of pancreatitis and 1 case of sepsis. All complications improved after symptomatic treatment. No biliary stent or ${ }^{125}$ I seed strip displacement was found in the postoperative reexamination. There was no significant difference in the incidence of complications between the two groups. See Table 3.

Table 3

complications in the two groups

\begin{tabular}{|llll|}
\hline Complication & Combination group $(\mathrm{n}=\mathbf{7 4})$ & Control group $(\mathrm{n}=\mathbf{8 1})$ & $\boldsymbol{p}$ \\
\hline Abdominal pain & 8 & 6 & 0.460 \\
\hline Bleeding & 1 & 0 & 0.294 \\
\hline pancreatitis & 2 & 2 & 0.927 \\
\hline cholangitis & 1 & 0 & 0.294 \\
\hline septicemia & 2 & 1 & 0.508 \\
\hline
\end{tabular}

\subsection{Stent opening time}

After $3,5,7,9$ and 12 months of follow-up or upon return to the hospital for reexamination, the patency of the biliary tract was examined. The overall opening times of the biliary stents in the two groups were $9.01 \pm 4.38$ months and $6.24 \pm 0.48$ months, respectively. Kaplan-Meier analysis was used to draw the survival curve (Fig. 3a), and the log rank test was used to compare them. The results showed that the 
opening time of biliary stents loaded with ${ }^{125}$ I seeds was significantly longer than that of simple biliary stents $(P<0.05)$.

\subsection{Survival and its influencing factors}

The cumulative survival rates of the two groups of patients at 3, 6 and 12 months were $99 \%, 89 \%$, and $68 \%$ (combined group) vs $96 \%, 79 \%$, and $47 \%$ (control group). The survival curves of the two groups were drawn by the Kaplan-Meier method (Fig. 3b). The log-rank test showed that the average survival time of the combined group and control group was $12.08 \pm 5.38$ months and $9.10 \pm 4.16$ months, respectively. There was a significant difference in survival time between the two groups $\left(\chi^{2}=10.576, p=0.001\right)$. Univariate analysis showed that the type of biliary obstruction, intrahepatic metastasis and stent type were significantly correlated with survival. Multivariate Cox analysis showed that intrahepatic metastasis and stent type were independent prognostic factors that affected survival (Table 4).

Table 4

Univariate and multivariate analyses of prognostic factors for survival

\begin{tabular}{|c|c|c|c|c|}
\hline \multirow[t]{2}{*}{ Variable } & \multicolumn{2}{|c|}{ Univariate Analysis } & \multicolumn{2}{|c|}{ Multivariate Analysis } \\
\hline & $\mathrm{HR}(95 \% \mathrm{Cl})$ & $\begin{array}{l}P \\
\text { Value }\end{array}$ & $\mathrm{HR}(95 \% \mathrm{Cl})$ & $\begin{array}{l}P \\
\text { Value }\end{array}$ \\
\hline $\operatorname{Sex}(M / F)$ & $\begin{array}{l}0.915(0.496- \\
1.427)\end{array}$ & 0.467 & & \\
\hline Age $(y)(\geq 50 /<50)$ & $\begin{array}{l}0.824(0.475- \\
1.543)\end{array}$ & 0.147 & & \\
\hline Bismuth type(I/II) & $\begin{array}{l}2.154(1.245- \\
3.517)\end{array}$ & 0.008 & $\begin{array}{l}2.067(1.159- \\
4.282)\end{array}$ & 0.074 \\
\hline KPS score $(\geq 70 /<70)$ & $\begin{array}{l}0.516(0.278- \\
1.162)\end{array}$ & 0.143 & & \\
\hline $\begin{array}{l}\text { Total bilirubin level }(\geq 200 /<200) \\
(\mu \mathrm{mol} / \mathrm{L})\end{array}$ & $\begin{array}{l}1.017(0.754- \\
2.143)\end{array}$ & 0.241 & & \\
\hline Intrahepatic metastasis $(\mathrm{Y} / \mathrm{N})$ & $\begin{array}{l}0.014(0.015- \\
0.212)\end{array}$ & $<0.001$ & $\begin{array}{l}0.021(0.004- \\
0.131)\end{array}$ & $<0.001$ \\
\hline lodine-125 Seeds(with/without) & $\begin{array}{l}3.287(2.112- \\
7.925)\end{array}$ & $<0.001$ & $\begin{array}{l}4.923(2.435- \\
8.568)\end{array}$ & $<0.001$ \\
\hline
\end{tabular}

\section{Discussion}

Most patients with obstructive jaundice caused by malignant tumors lose the opportunity for surgical treatment[12]. Biliary stent implantation and reopening of the occluded bile duct is the main treatment method at present. Tumor progression and stent intimal hyperplasia are the main reasons for the recurrence or deterioration of jaundice in patients[13-14]. Therefore, how to control tumor growth and delay the hyperplasia of the bile duct intima is the key to preventing stent blockage and prolonging the 
survival time of patients. Previous clinical studies have used biliary stents combined with external radiotherapy[15], biliary stents combined with arterial intubation chemoembolization[16] and the installation of radiotherapy devices for biliary radiotherapy[17] and other methods. Due to the poor basic condition of the patient, the sensitivity of surrounding organs to radiotherapy, the limitation of the primary tumor type and the cumbersome surgical operation, etc., it cannot be widely used in clinical applications. SEMS combined with ${ }^{125}$ I seed implantation and intracavitary irradiation has been used to treat malignant biliary obstruction[18-19], and certain clinical effects have been achieved.

${ }^{125}$ I seeds are a kind of low-dose-rate microradiation that release $y$-rays. Because of their short range of radiation, low penetration, and maximum killing of tumors without damaging the surrounding normal tissues, ${ }^{125}$ I seeds are widely used in radiotherapy. They have been applied to the clinical treatment of prostate cancer, rectal cancer, nasopharyngeal cancer, liver cancer and other malignant tumors[20-21] and have achieved good results. Based on the advantages of ${ }^{125} \mathrm{I}$ seeds, we explored the difference in clinical efficacy between SEMS combined with ${ }^{125}$ I seeds and SEMS alone in the treatment of MBO. In this study, first, ${ }^{125}$ I seeds were placed in the carrier device and fixed on the bile duct wall through the support of a biliary stent, which effectively solved the difficulty of direct puncture and implantation of ${ }^{125}$ I seeds for the treatment of malignant tumors growing along the bile duct. Compared with simple biliary stent implantation, a biliary stent combined with ${ }^{125} \mathrm{I}$ seed implantation will not cause obstruction of bile drainage and will ensure the patency of the biliary tract. The continuous radiation can effectively inhibit and kill tumor cells and reduce the proliferation of biliary intima. This treatment has an obvious curative effect in delaying the recurrence of obstructive jaundice. In this study, the bilirubin level was significantly improved at 1 week and 1 month after biliary stent implantation in each group $(P<0.001)$. The two methods had good short-term curative effects in the treatment of MBO, and the effect on jaundice was obvious, but the difference between the two groups was not statistically significant $(P>0.05)$. Even though the two groups had no significant difference in short-term jaundice reduction, the recurrence time of jaundice in the combined group was significantly longer than that in the control group (9.01 mo. vs. 6.79 mo., $P<0.001)$. This result is comparable to previous results[22-23]. This study also found that the incidence of jaundice recurrence started to differ between groups 5 months after biliary stent implantation. The main reason was that the combination method effectively killed or inhibited the tumor cells in the bile duct wall and then inhibited the intimal hyperplasia around the biliary stent and thereby delayed the recurrence of obstructive jaundice. In addition to delaying the recurrence of jaundice, a biliary stent combined with brachytherapy can also prolong the survival time of patients. Although Isayama et al.[24] reported that the cumulative survival rate of a biliary stent combined with internal radiotherapy was no different from that of R1 resection, the median survival time of the stent combined with internal radiotherapy group was significantly longer than that of the stent-only group. The results of this article also show that the median survival time of the combined group was significantly longer than that of the control group (12.08 mo. vs. 9.10 mo., $P<0.001)$.

In addition, existing studies have shown that the incidence of early complications of percutaneous biliary stent implantation is $5.7-28 \%$, the mortality rate related to surgery is $0-4 \%$, and the mortality rate 1

Page $12 / 19$ 
month after surgery is $9-15 \%$. most of the complications can be resolved by conservative medical treatment, and the occurrence of death one month after surgery is generally related to some of the original underlying diseases of the patient[25]. The results of this article show that the early complication rates of the two groups of patients were $18.92 \%$ and $11.11 \%$, both of which improved after conservative medical treatment, and the surgery-related mortality rate was $0 \%$. Previous studies have shown that biliary drainage brings a risk of biliary tract infection and puncture tumor implantation and metastasis, so percutaneous biliary drainage and stent implantation should be done with cautious[26]. Our study did not observe the occurrence of tumor implantation or metastasis in the puncture tract, which was due to the improvements of interventional treatment technology and equipment in recent years. Therefore, percutaneous biliary stent combined with ${ }^{125}$ I seed implantation is a relatively safe and feasible palliative treatment.

In conclusion, percutaneous liver puncture biliary stent placement combined with ${ }^{125}$ I seed implantation is safe, effective and feasible for patients with unresectable Bismuth type I or II malignant biliary obstruction. This treatment scheme can extend the patency time of the stent as well as the survival time of patients. The limitations of this study are that it was retrospective, and the sample size was small. Therefore, prospective multicenter and larger randomized controlled studies are needed to further confirm our results.

\section{Abbreviations}

PTCD: Percutaneous Transhepatic Cholangial Drainage; SEMS: Self-expandable Metallic Stent; MBO: Malignant Biliary Obstruction; TPS:Treatment Planning System; ECOG:Eastern Cooperative Oncology Group; CT: computed tomography; MRCP: Magnetic Resonance Cholangiopancreatography; ECG: Electrocardiogram

\section{Declarations}

\section{Acknowledgments}

We would like to thank The biliary stent was provided by the Nanjing Institute of Minimally Invasive Research, China

\section{Authors' contributions}

Conceptualization: Shao-Bao Xu,Zong-Gen Gao ,Chang-Sheng Shi ,Ming-Xue Su. Data curation:Zong-Gen Gao, Chang-Sheng Shi ,Ming-Xue Su. Formal analysis: Kai-Cai Liu,Wei-Fu Lv. Funding acquisition: Wei-Fu Lv,Dong Lu. Investigation: Wei-Fu Lv,Dong Lu. Methodology: Chun-Ze Zhou,De-Lei Cheng. Software: ChunZe Zhou. Validation: Wei-Fu Lv, Chun-Ze Zhou. Visualization: Wei-Fu Lv, De-Lei Cheng. Writing-original draft:Kai-Cai Liu. Writing-review and editing: Kai-Cai Liu,Wei-Fu Lv.

\section{Funding}


This study was supported by the Fundamental Research Funds for the Central Universities (No. WK9110000061).

\section{Availability of data and materials}

The datasets used and/or analyzed during the current study are available from the corresponding author on reasonable request.

\section{Declarations Ethics approval and consent to participate}

Not applicable.

\section{Consent for publication}

Not applicable.

\section{Competing interests}

The authors declare that they have no competing interests.

\section{References}

1. Fernandez $Y$, Viesca M, Arvanitakis M. Early Diagnosis And Management Of Malignant Distal Biliary Obstruction: A Review On Current Recommendations And Guidelines. Clin Exp Gastroenterol. 2019;12:415-32.

2. Harsha Moole, Matthew Bechtold, Srinivas R Puli. Efficacy of preoperative biliary drainage in malignant obstructive jaundice: a meta-analysis and systematic review. World J Surg Oncol. $2016 \mathrm{Jul}$ 11;14(1):182.

3. Boulay BR, Birg A. Malignant biliary obstruction: From palliation to treatment. World J Gastrointest Oncol. 2016;8(6):498-508.

4. Zhou C, Li H, Huang Q, Wang J. Gao K. Biliary self-expandable metallic stent combined with lodine125 seeds strand in the treatment of hilar malignant biliary obstruction[J]. The Journal of international medical research, 2019:030006051988784.

5. Beleites E, Neupert G, Augsten G. , Vogel W,Schubert H. Endoscopic biliary stenting: indications, choice of stents, and results: European Society of Gastrointestinal Endoscopy (ESGE) Clinical Guideline - Updated October 2017[J]. Endoscopy.2018 Sep;50(9):910-930.

6. Mukai S, Itoi T, Baron TH., Takada T, Strasberg SM, Pitt HA,et al. Indications and techniques of biliary drainage for acute cholangitis in updated Tokyo Guidelines 2018[J]. J Hepatobiliary Pancreat, 2017.2017 Oct;24(10):537-549.

7. Hasimu A, Gu JP, Ji WZ, ,Zhang HX, Zhu DW, Ren WX. Comparative Study of Percutaneous Transhepatic Biliary Stent Placement with or without lodine-125 Seeds for Treating Patients with 
Malignant Biliary Obstruction[J]. Journal of Vascular Interventional Radiology Jvir. 2017;28(4):58393.

8. Kim JY, Ko GB, Lee TH, Park SH, ,Kim SJ. Partially covered metal stents may not prolong stent patency compared to uncovered stents in unresectable malignant distal biliary obstruction. Gut Liver 2017; 11: 440-446.

9. Won JH, Lee JD, Wang HJ, Han JH, Kim JH, Kim KH. et al. Effects of a holmium-166 incorporated covered stent placement in normal canine common bile ducts.J Vasc Interv Radiol. 2005 May;16(5):705-11.

10. Zhou WZ, Fu YM, Yang ZQ, Shi HB, Liu S, Xia JG., et al.Study of Percutaneous Stent Placement with lodine-125 Seed Strand for Malignant Biliary Obstruction[J]. Cardiovasc Intervent Radiol. 2019 Feb;42(2):268-275.

11. Li Shuangxi, Li Baohua, Li Lei, Yang Xujun, Xu Fangyu. Wenhui Wang.The efficacy of the combination of percutaneous transhepatic biliary drainage and 125 I stranded seeds for malignant bile duct obstruction treatment[J].J Contemp Brachytherapy.2020 Jun;12(3):225-232.

12. Michael Fernandez Y Viesca,Marianna Arvanitakis. Early Diagnosis And Management Of Malignant Distal Biliary Obstruction: A Review On Current Recommendations And Guidelines. Clin Exp Gastroenterol. 2019 Nov 5;12:415-43.

13. Almadi MA. Barkun A, Martel M. Plastic vs. Self-Expandable Metal Stents for Palliation in Malignant Biliary Obstruction: A Series of Meta-Analyses. Am J Gastroenterol. 2017;112:260-73.

14. B Acu, E Kurtulus Ozturk. Feasibility and safety of percutaneous transhepatic endobiliary radiofrequency ablation as an adjunct to biliary stenting in malignant biliary obstruction.Diagn Interv Imaging. 2018 Apr;99(4):237-245.

15. Takamura A, Saito H, Kamada T,Hiramatsu K,Takeuchi S, Hasegawa M,et al. Intraluminal low-doserate 192Ir brachytherapy combined with external beam radiotherapy and biliary stenting for unresectable extrahepatic bile duct carcinoma.[J]. International Journal of Radiation Oncology Biology Physics, 2003, 57(5): 1357-1365.

16. Guangshao Cao, Huicun Cao, Jian Liu.One-channel double stent implantation for hilar biliary obstructions.Exp Ther Med. 2013 Apr;5(4):1179-1183.

17. Xinjian Xu, Jingjing Li, Juan Wu ,Rui Zhu,Wenbin Ji. A Systematic Review and Meta-analysis of Intraluminal Brachytherapy Versus Stent Alone in the Treatment of Malignant Obstructive Jaundice.Cardiovasc Intervent Radiol. 2018 Feb;41(2):206-217.

18. Pang Q, Zhou L, Hu XS, ,Wang Y,Liu HCl. Biliary stenting alone versus biliary stenting combined with 125 I particles intracavitary irradiation for the treatment of advanced cholangiocarcinoma[J].Sci Rep. 2019 Aug 5;9(1):11348.

19. Wang T, Liu S, Zheng YB, Song XP, Sun BL, Jiang WJet al. Clinical Study on Using 125 I Seeds Articles Combined with Biliary Stent Implantation in the Treatment of Malignant Obstructive Jaundice.Anticancer Res. 2017 Aug;37(8):4649-4653. 
20. Lu J, Guo JH, Zhu HD, Zhu JY, Chen L,Teng GJ. Safety and efficacy of irradiation stent placement for malignant portal vein thrombus combined with transarterial chemoembolization for hepatocellular carcinoma: a single-center experience. J Vasc Interv Radiol. 2017;28:786-794.e3

21. Huo X, Wang H, Yang J, Li X, Yu ZT. Effectiveness and safety of CT-guided (125)I seed brachytherapy for postoperative locoregional recurrence in patients with non-small cell lung cancer. Brachytherapy. 2016;15:370-80.

22. Lu J, Guo JH, Zhu HD. et al. Palliative treatment with radiation-emitting metallic stents in unresectable Bismuth type III or IV hilar cholangiocarcinoma[J]. Esmo Open. 2017;2(4):e000242., Zhu G Y, Wang Y, Zhang Q, Chen L .

23. Zhu H, Guo J, Huang M. et al. Irradiation stents vs. conventional metal stents for unresectable malignant biliary obstruction: A multicenter trial[J]. J Hepatol. 2018 May;68(5):970-7., Ji J, Xu H, Lu $\mathrm{J}$.

24. Isayama H sujino, Nakai T. Y,Sasaki T,Nakagawa K,Yamashita H,et al. Clinical benefit of radiation therapy and metallic stenting for unresectable hilar Cholangiocarcinoma[J]. World $\mathrm{J}$ Gastroenterol.2012,18:2364-2370.

25. Inal $M$, Akgul E, Aksungur E,Demiryürek $H, O ̈$. Yağmur. Percutaneous self-expandable uncovered metallic stents in malignant biliary obstruction. Complications, follow- up and reintervention in 154 patients [J]. Acta Radiol,2003,44: 139-146.

26. Wee Ngu,Michael Jones,Chrisopher P Neal.Preoperative biliary drainage for distal biliary obstruction and post-operative infectious complications.ANZ J Surg. 2013 Apr;83(4):280-6.

\section{Figures}




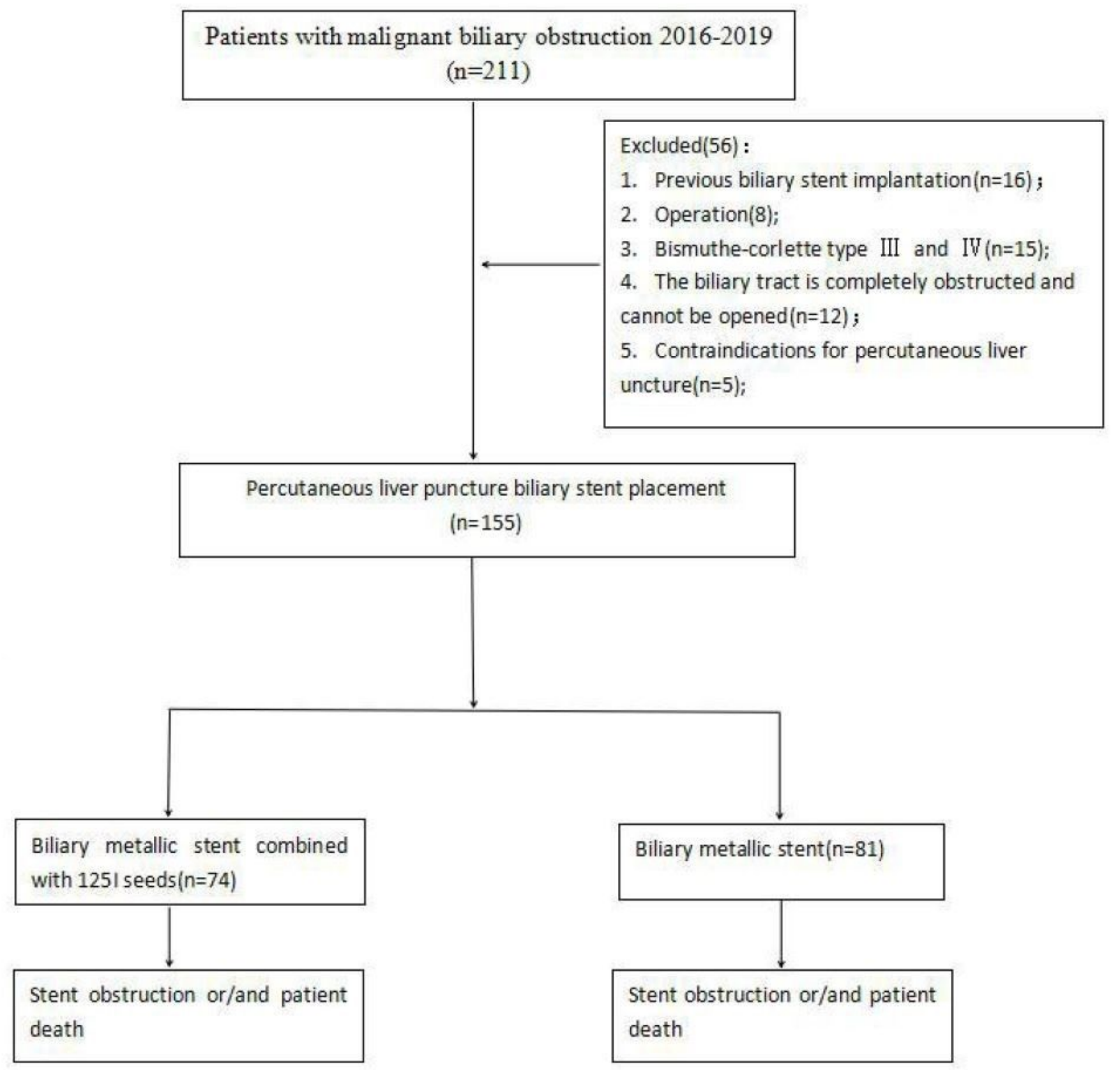

\section{Figure 1}

Flow diagram illustrating the treatment process. 


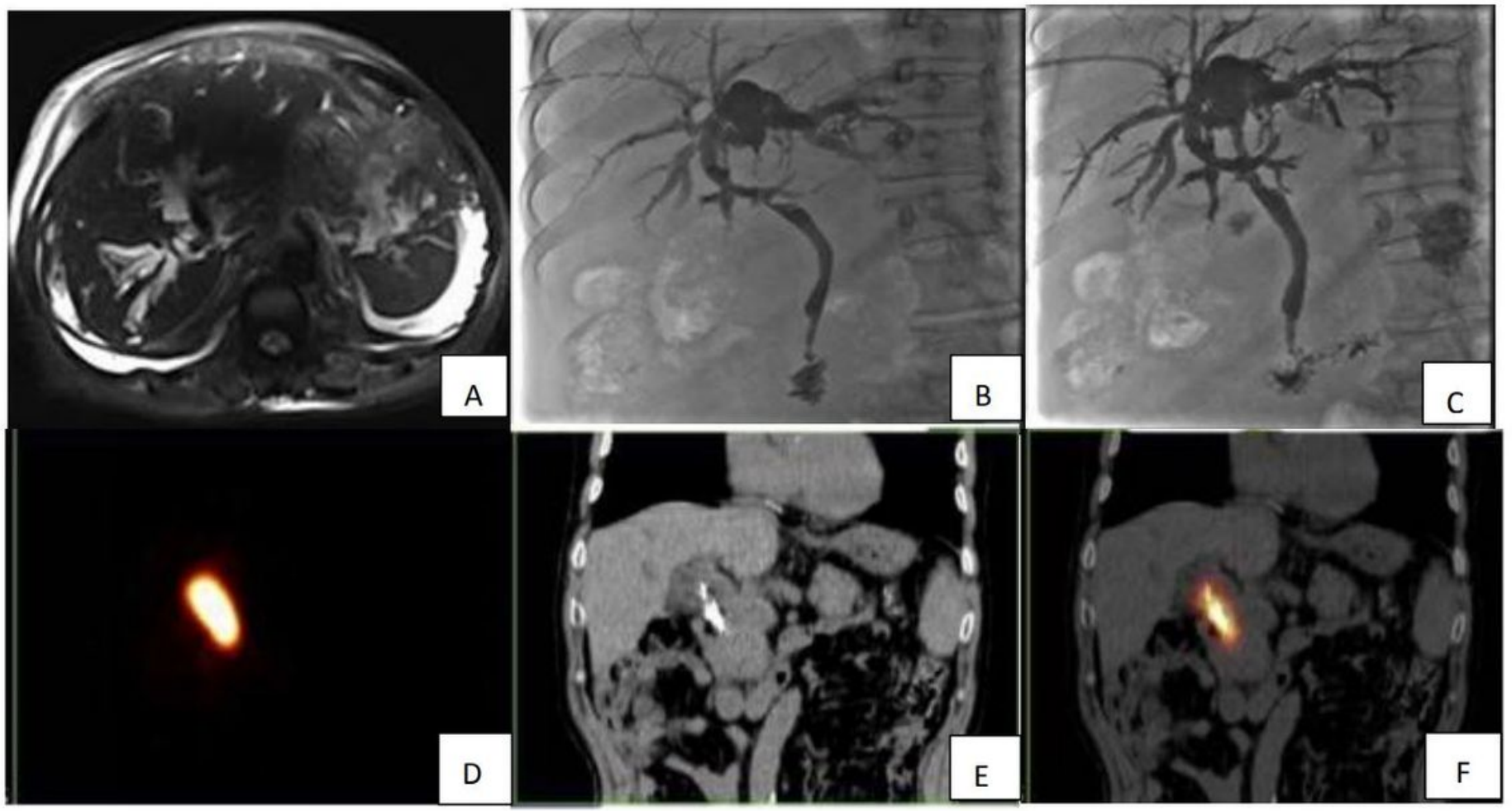

Figure 2

A 66-year-old man with cholangiocarcinoma. Preoperative MR scan showed intrahepatic bile duct dilatation (A), and DSA showed obstruction of the middle and upper segments of the common bile duct with intrahepatic bile duct dilatation (B). After implantation of the biliary stent combined with $125 \mathrm{I}$ seeds, the obstruction was relieved, and the contrast agent entered the intestinal cavity smoothly (C). Postoperative SPECT/CT showed that the gamma radiation range of iodine particles covered the lesions in the middle and upper common bile duct (D-F). 


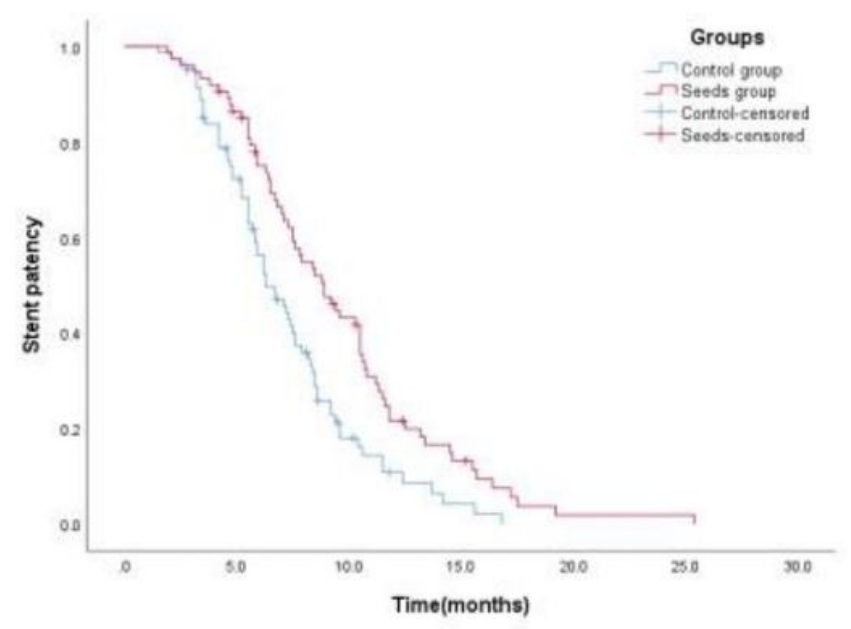

a

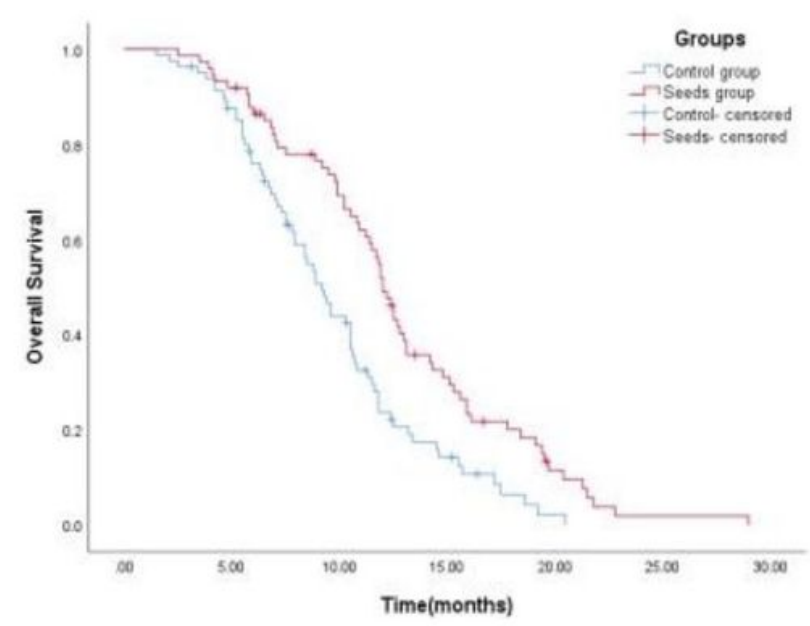

b

Figure 3

(a) Kaplan Meier survival curves for comparison of stent patency time between combined group and control group $(P=0.001)$. (b) Kaplan-Meier survival curves for comparison of OS between combined group and control group $(P<0.001)$ 